\title{
Fall prevention in the elderly population
}

Background and epidemiology: A quick visit to the Quick Stats section on the Canadian Institute of Health Information Web site (http://secure.cihi.ca/cihi web/splash.html) stopped us short: falls accounted for $85 \%$ of the 73113 injury-related admissions among people over 65 years old in the year 1999/2000 (Fig. 1).

The impact of falls on our elderly population and health care system is huge, resulting in debilitating injuries, loss of independence and often death or transfer to an institution. Estimates suggest that about 1 in 3 elderly people living in the community will experience at least one fall a year. This multifaceted public health problem is by no means uniquely Canadian. Many countries populated by aging baby boomers are experiencing and anticipating increasing numbers of injuries from falls and are developing compre-

$\vec{\infty}$ hensive prevention strategies to $\neq$ reduce this burden.

Falls are the result of a complex combination of medical and lifestyle factors operating alone or in conjunction with precipitating environmental factors. Common modifiable risk factors

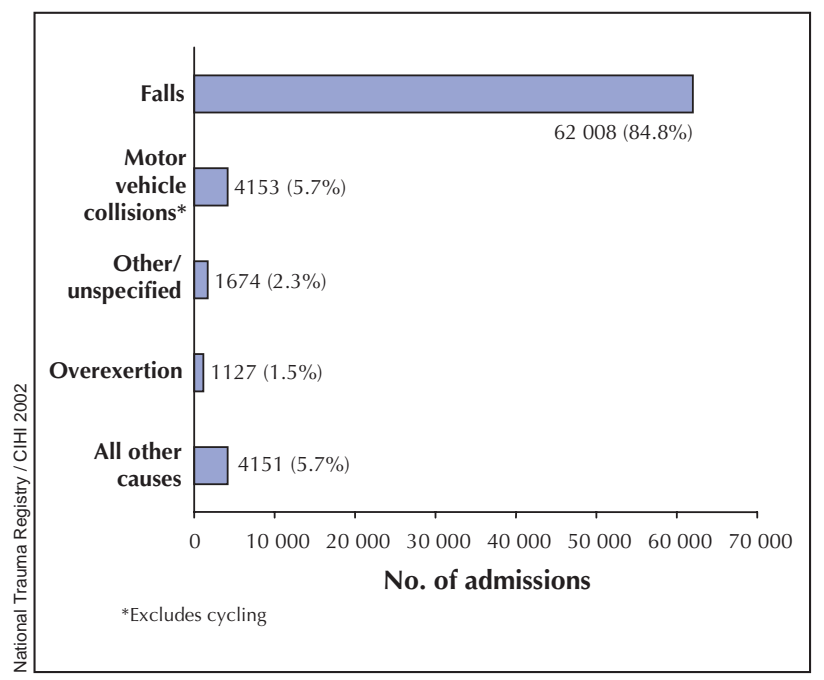

Fig. 1: Causes of injury-related admissions among people $>65$ years old in Canada, 1999/2000. include muscle weakness, problems with balance, gait or stability, multiple drug therapy, postural hypotension and cardiac disorders. $^{2}$

Clinical management: Physicians can play a major role in identifying elderly patients at risk of falling. At least annually, during routine medical visits, they should ask every older patient about the occurrence of falls and any gait or balance problems. A quick screening tool is the "get up and go test," which involves looking for unsteadiness as the patient gets up from a chair without using his or her arms, walks a few metres and returns. Also useful is the Tinetti Assessment Tool, ${ }^{3}$ which uses a 28 -point scale to assess specific components of gait and balance (available online at www.cmaj.ca/cgi/content /full/171/7/724/DC1). In general, scores of 19 to 24 indicate a risk for falls, with scores below 19 indicating a high risk.

Patients with problems detected by the screening tools require a formal assessment, including determination of their history of falls, circumstances surrounding the falls, use of medications, history of illnesses and mobility problems, evaluation of gait and balance, orthostatic blood pressure and pulse, and ophthalmological, neurological and cardiovascular examinations. ${ }^{2}$

Physicians should encourage older patients to adopt a lifestyle to maintain their balance through regular exercise, healthy eating and responsible drinking, and make appropriate and timely referrals to allied health care workers (e.g., physiotherapists) to assist with footwear and mobility aids.

Prevention: Patients with a history of falls can benefit from a directed assessment and modification of environmental hazards (e.g., problems with building design, handrails, surfaces, changes in elevation and lighting). In many communities, occupational therapists or public health agencies are available to complete these assessments. The reduction of hazards at home will reduce the risk of falls, especially when combined with strategies to improve elderly people's confidence and decrease their fear of falling, such as exercise programs to improve balance and muscle strength.

In addition to identifying people at risk of falls, effective prevention involves modifying their risk factors where possible. Multifaceted strategies that target a variety of risk factors hold promise for reducing the incidence of falls among people at high risk and are considered to be a good investment. ${ }^{2}$ Specific interventions might include the development of policies to prevent falls in long-term care facilities and public places and the implementation of communitybased strategies that combine exercise programs to improve balance and muscle strength with education sessions on how to prevent falls. The key is to identify people at risk of falling and to refer them to local programs or resources.

\section{Erica Weir}

Associate Medical Officer of Health

Luana Culmer

Public Health Nurse, Adult Health

Kingston, Frontenac and Lennox

\& Addington Health Unit

Kingston, Ont.

\section{References}

1. Van Beurden E, Kempton A, Sladden $\mathrm{T}$, Garner E. Designing an evaluation for a multiple-strategy community intervention: the North Coast Stay on Your Feet program. Aust N Z 7 Public Health 1998;22:115-9.

2. Tinetti ME. Preventing falls in elderly persons. $N$ Engl 7 Med 2003;348 (1):42-9.

3. Tinetti ME. Performance-oriented assessment of mobility problems in elderly patients. 7 Am Geriatr Soc 1986;34:119-26. 